\title{
STABILITY ANALYSIS IN THE RESTRICTED FOUR BODY PROBLEM WITH OBLATNESS AND RADIATION PRESSURE.
}

\author{
M.N. Ismail ${ }^{1}$, Sahar H. Younis ${ }^{2 *}$, Ghada F. Mohamdien \\ 1 Astonomy and Meteorology Department, Faculty of Science, Al-Azhar University, Egypt \\ 2 Math. Dep., Faculty of Science (Girls), Al-Azhar University, Egypt \\ 3 National Research Institute of Astronomy and Geophysics (NRIAG), Egypt \\ *Correspondence: d.sahar92@azhar.edu.eg
}

\begin{abstract}
In the present work, the canonical form of the differential equations is derived from the Hamiltonian function $\mathrm{H}$ which is obtained for the system of the four-body problem. This canonical form is considered as the equations of motion, the equilibrium points of the restricted four-body problem are studied under the effects of radiation pressure and oblatness Lyapunov function is used to provide a method for showing that equilibrium points are stable or asymptotically stable. If the system has an equilibrium point conditionally the eigenvalues of the system contain negative real parts, the scalar potential function is positive definite, then The Lyapunov center's theorem is used to analyze the stability and periodicity of the motion of orbits about these equilibrium points of the restricted four-body problem. From this theorem, the Lyapunov function is found. Also, the stability regions are studied by using The Poincare maps, an analytical and numerical approach had been used. A cod of Mathematica is constructed to truncate these steps. The periodic orbits around the equilibrium points are investigated for the Sun-Earth-Moon system.
\end{abstract}

Keywords: The Restricted Problems, The Liberation Points, Oblatness, Radiation Pressure Effects, Stability about Libration Points.

\section{INTRODUCTION}

Many authors have studied the four-bodyproblem in astrodynamics. Several attempts to model the problem in a mathematical form using classical treatment or Hamiltonian treatment. As seen in [1], [2] and [3] the motion of spacecraft in Earth-Moon-Sun system is studied in which the main two primaries Earth and Moon are revolved in circular orbits around their barycenter, while the perturbing body Sun is moved in a circular orbit around the central masses of the Earth-Moon-Sun system. [4],[5] and [6] considered Sun, Earth, and Moon as distinct particles of finite masses. In [7] the author illustrated the collinear equilibrium points into the phase space in the vicinity of the Sun-perturbed Earth-Moon by using the quasibi-circular model. In [8] the author determined the periodic motion in the vicinity of the Sunperturbed Earth-Moon triangular points (L4 and L5) by using the restricted Hill four-body model. Moreover, he examined the invariant manifolds associated with these solutions. The authors in [9], [10], [11] and [12] studied the restricted four-body problem as equal masses located at the vertices of an equilateral triangle, also, zero velocity curves, the equilibrium points, and families of periodic orbits were was studied. In [13] and [14] the authors proved the stability, periodic orbits around the triangular points of the Earth-Moon system taken into account a perturbation by the Sun. Moreover, a periodic solution for the Sun-Earth-Moon system using numerical integration is obtained. In the papers [15] and [16] authors generated periodic and quasi-periodic orbits using the numerical technique of Poincare surface sections. The paper [17] author found the Lissajous orbits and Lyapunov orbits around the collinear points in the restricted three-body problem under the effect of oblateness. In [18] the authors studied equilibrium points and zero velocity surfaces in the restricted four-body problem with solar wind drag. The authors [19] and [20] put a model for the sun's radiation effect on restricted four bodies.[21] the author used Lyapunov's center theorem to study the existence of periodic orbits families about the equilibrium points of a dynamical system with $\mathrm{n}$ degrees of freedom described 
by a real analytical Hamiltonian $\mathrm{H}\left(\mathrm{x}_{1}, \mathrm{x}_{2}, \ldots, \mathrm{x}_{\mathrm{n}}\right)$. The authors [22] and [23] considered the origin $x_{i}=0$ of the phase space as an elliptic equilibrium and the eigenvalues of this system are calculated by using the Lyapunov function.

\section{The direct method of Lyapunov}

The direct method of Lyapunov 's (also called the second method of Lyapunov) using to determine the stability of a system without explicitly integrating the differential equations. The idea of this method is a generalization "measure of energy" in a system. Then the rate of change of the energy of the system to certain stability can be studied.

\section{Definition of a quadratic form.}

Let $\mathrm{A}$ denote an $\mathrm{n} \times \mathrm{n}$ symmetric matrix with real entries and let $x$ denote an n column vector. Then $\mathrm{Q}=\mathrm{x} \boldsymbol{A} \boldsymbol{x}$ is said to be a quadratic form.

Classification of the quadratic form $\mathrm{Q}=\mathrm{x} \boldsymbol{A} \boldsymbol{x}$ :

A quadratic form is said to be:

a: negative definite: $\mathrm{Q}<0$ when $\mathrm{x} \neq 0$

b: negative semi-definite: $Q \leq 0$ for all $x$ and $\mathrm{Q}=0$ for some $\mathrm{x} \neq 0$.

c: positive definite: $\mathrm{Q}>0$ when $\mathrm{x} \neq 0$

$\mathrm{d}$ : positive semi-definite: $\mathrm{Q} \geq 0$ for all $\mathrm{x}$ and $\mathrm{Q}=0$ for some $\mathrm{x} \neq 0$

e: positive indefinite: $\mathrm{Q}>0$ for some $\mathrm{x}$ and $\mathrm{Q}<0$ for some other $\mathrm{x}$ [24],[25],[26] and [27].

\section{Condition of stability of the Lyapunov} Function

1- Positive-definite:

$\mathrm{V}(\mathrm{x})>0, x \neq 0, v(0)=0$

2- Decreasing (Or non- increasing) along the trajectories of the system.

3- Derivatives negative (or semi-definite)

$\frac{\mathrm{dV}(\mathrm{x}(\mathrm{t}))}{\mathrm{dt}}=\frac{\partial \mathrm{V}(\mathrm{x}(\mathrm{t}))}{\partial \mathrm{x}} \mathrm{x}<0, \quad \boldsymbol{x} \neq \mathbf{0}$.
Where $x(t)$ is the equilibrium point for the system.

Theorem: the matrix $\mathbf{A}$ is Hurwitz if and only if for any $Q=Q^{T}>\mathbf{0}$ there is $\mathrm{p}=\mathrm{p}^{\mathrm{T}}>\mathbf{0}$ that satisfies the Lyapunov equation

$$
\mathrm{pA}+\mathrm{A}^{\mathrm{T}} \mathrm{p}=-\mathrm{Q}
$$

Moreover, if $\mathbf{A}$ is Hurwitz, then $\mathbf{P}$ is the unique solution [28] and [29].

\section{Lyapunov Stability Theorem}

1- Given a positive definite function $V(x)$

2- If the derivatives of $V(x)$ along the trajectories of the system is

a) negative semidefinite, the equilibrium is stable in the sense of Lyapunov.

b) negative definite, the equilibrium is asymptotically stable.

c) positive definite, the equilibrium is unstable.[30]

In this work, it is considered that three bodies are moving in the same plane, while the fourth body is acting as a perturbing force. So that some important restrictions must be taken into account:

i. The gravitation and radiation forces are effects on the fourth body.

ii. The three masses specify that $\mathrm{m}_{1}>\mathrm{m}_{2}>>\mathrm{m}$, and $\mathrm{m}$ is considered as the test particle, all the three masses are moving in the same plane.

iii. The masses $\mathrm{m}_{1}$ and $\mathrm{m}_{2}$ are considered as primaries and the mass ratio $\mu=\frac{m_{2}}{m_{1}+m_{2}}$ is used, and ميو3 referred to the dimensionless of the sun. The distance between $\mathrm{m}_{1}$ and $\mathrm{m}_{2}$ is considered as a unity.

iv. The mass $\mathrm{m}_{1}$ is considered as an oblate body with oblatness coefficient $A=\frac{R_{e}^{2}-R_{p}^{2}}{S R}, R_{e}$ is the equatorial and $R_{P}$, the polar radii and $\mathrm{R}$ is the separation distance between the two primaries.

\section{Equations of motion:}


Fig. 1 illustrates the description of the problem where the barycenter is the origin of this system.

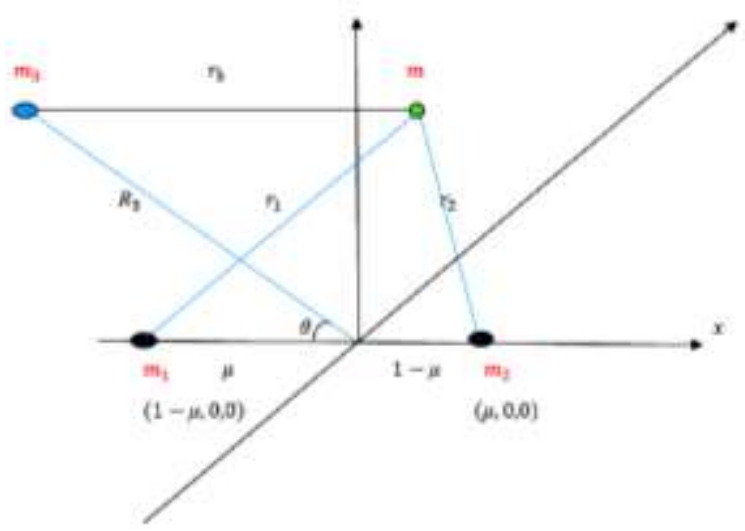

Fig 1: the description of the four-body problem

Now, the effect of $m_{3}$ can be evaluated as:

$\mathrm{F}=\mathrm{F}_{\mathrm{g}}-F_{\mathrm{r}}=\mathrm{F}_{\mathrm{g}}\left(1-\frac{\mathrm{F}_{\mathrm{r}}}{\mathrm{F}_{\mathrm{g}}}\right)=(1-\beta) \mathrm{F}_{\mathrm{g}}$

Where $\mathrm{F}$ is resultant force, and $\mathrm{F}_{\mathrm{g}}, \mathrm{F}_{\mathrm{r}}$ are the gravitation and radiation forces respectively, $\beta$ is the radiation pressure coefficient. Since the potential of the force is defined as $U=-\int F$ dr , then the potential of the problem can be written as

$V=\frac{1-\mu}{r_{1}}+\frac{1-\mu}{r_{2}}+\frac{(1-\beta) \mu_{3}}{r_{3}}+\frac{A(1-\mu)}{r_{1}}$

where

$r_{1}=\sqrt{(x-(1-\mu))^{2}+y^{2}+z^{2}}$

$r_{2}=\sqrt{(x-\mu)^{2}+y^{2}+z^{2}}$

$r_{3}=\sqrt{\left(x-R_{3} \cos \theta\right)^{2}+\left(y-R_{3} \sin \theta\right)^{2}+z^{2}}$

Now, the system has a Lagrangian function represented by $\mathrm{L}=\mathrm{T}-\mathrm{V}$, where $\mathrm{T}$ is the total kinetic energy and Vis the total potential energy of the system. The kinetic energy is obtained from the motion of the mass $\mathrm{m}$, and the potential is due to the effects of the other three bodies, then

$\mathrm{L}=\mathrm{T}-\mathrm{V}=\frac{1}{2\left((\dot{\mathrm{x}}-\mathrm{y})^{2}+(\dot{\mathrm{y}}+\mathrm{x})^{2}+\dot{\mathrm{z}}^{2}\right)}-V$

The Hamiltonian is defined as:

$$
\mathrm{H}=\mathrm{P}^{\mathrm{T}} \dot{x}-L
$$

Where:

$$
P=\left[\begin{array}{l}
p_{x} \\
p_{y} \\
p_{z}
\end{array}\right]
$$

Where $p_{x}, p_{y}$, and $P_{z}$ are the momenta components, then substitute from Equation (2), and (4) into Equation (5), then the Hamiltonian is obtained:

$$
\begin{aligned}
& \mathrm{H}=\frac{1}{2}\left(\mathrm{p}_{\mathrm{x}}^{2}+\mathrm{p}_{\mathrm{y}}^{2}+\mathrm{p}_{\mathrm{z}}^{2}\right)-\left(\mathrm{p}_{\mathrm{x}} \mathrm{y}-\mathrm{p}_{\mathrm{y}} \mathrm{x}\right) \\
& -\left(\frac{1-\mu}{\mathrm{r} 1}+\frac{\mu}{\mathrm{r} 2}+\frac{(1-\beta) \mu 3}{\mathrm{r} 3}+\frac{\mathrm{A}(1-\mu)}{2 \mathrm{r} 1^{3}}\right)
\end{aligned}
$$

And the canonical form is given by:

$$
\dot{\mathrm{X}}=\frac{\partial \mathrm{H}}{\partial \mathrm{P}}
$$

$$
\dot{\mathrm{P}}=-\frac{\partial \mathrm{H}}{\partial \mathrm{X}}
$$

$$
\text { Where } \dot{\mathrm{X}}=\left[\begin{array}{l}
\dot{\mathrm{x}} \\
\dot{\mathrm{y}} \\
\dot{z}
\end{array}\right] \quad \text { and } \dot{\mathrm{P}}=\left[\begin{array}{l}
\dot{\mathrm{p}}_{\mathrm{x}} \\
\dot{\mathrm{p}}_{\mathrm{y}} \\
\mathrm{p}_{\mathrm{z}}
\end{array}\right]
$$

The system of Eqs. (8) is considered as the equations of motion.

\section{Libration Points:}

The libration points are given by setting $\dot{\mathrm{X}}=0$ and $\dot{\mathrm{P}}=0$, using Eqs (8), then

$$
\begin{gathered}
\frac{\partial \mathrm{H}}{\partial \mathrm{p}_{\mathrm{x}}}=\mathrm{p}_{\mathrm{x}}+\boldsymbol{y}=\mathbf{0} \\
\frac{\partial \mathrm{H}}{\partial \mathrm{p}_{\mathrm{y}}}=\mathrm{p}_{\mathrm{y}}-\mathrm{x}=\mathbf{0}
\end{gathered}
$$


$\frac{\partial \mathrm{H}}{\partial \mathrm{p}_{\mathrm{z}}}=\mathrm{p}_{\mathrm{z}}=\mathbf{0}$

And

$\frac{\partial \mathrm{H}}{\partial \mathrm{x}}=-\mathrm{p}_{\mathrm{y}}+\frac{\partial \mathrm{V}}{\partial \mathrm{x}}=\mathbf{0}$

$\frac{\partial H}{\partial y}=p_{x}+\frac{\partial V}{\partial y}=0$

$\frac{\partial \mathrm{H}}{\partial \mathrm{z}}=\frac{\partial V}{\partial \mathrm{z}}=0$

Since the three bodies moves in the $\mathrm{x}-\mathrm{y}$ plane, then $\mathrm{z}=0$ and $\mathrm{P}_{\mathrm{z}}=0$.

Then it is better to use the potential,

$$
\mathrm{U}(\mathrm{x}, \mathrm{y})=\frac{1}{2}\left(\mathrm{x}^{2}+y^{2}\right)-V(\mathrm{x}, \mathrm{y})
$$

From which

$$
\begin{aligned}
& \frac{\partial \mathrm{U}}{\partial \mathrm{x}}=\boldsymbol{x}-\frac{\partial \mathrm{V}}{\partial \mathrm{x}} \\
& \frac{\partial \mathrm{U}}{\partial \mathrm{y}}=\boldsymbol{y}-\frac{\partial \mathrm{V}}{\partial \mathrm{y}}
\end{aligned}
$$

From Eq. (12.1) by $\frac{\partial U}{\partial x}=0$, this will give the collinear libration points, while by solving Eqs. (12) for (x, y), this will give the non- collinear libration points.

\section{Analysis of Stability}

Now, it is needed to study the stability of each of the libration points obtained. Let

$$
\mathrm{Z}=\left[\begin{array}{l}
\mathrm{x} \\
\mathrm{y} \\
\mathrm{p}_{\mathrm{x}} \\
\mathrm{p}_{\mathrm{x}}
\end{array}\right]
$$

We can write the Hamiltonian system as:

$$
\dot{\mathrm{Z}}=\mathrm{J} \nabla_{\mathrm{z}} H
$$

Where $\mathbf{J}$ is the asymptotic matrix,

$$
\mathrm{J}=\left[\begin{array}{cc}
\mathbf{0}_{\mathrm{n} \times \mathrm{n}} & \mathrm{I}_{\mathrm{n} \times \mathrm{n}} \\
-\mathrm{I}_{\mathrm{n} \times \mathrm{n}} & \mathbf{0}_{\mathrm{n} \times n}
\end{array}\right]
$$

To study the motion near the libration point let

$$
\begin{aligned}
& \mathrm{q} 1=\mathrm{x}-\xi 1 \\
& \mathrm{q} 2=\mathrm{y}-\xi 2 \\
& \mathrm{p} 1=\mathrm{p}_{\mathrm{x}}+\xi 2 \\
& \mathrm{p} 2=\mathrm{p}_{\mathrm{y}}-\xi 1
\end{aligned}
$$

Where $\mathrm{q} 1$ and $\mathrm{q} 2$ are the new coordinates, p1 and $\mathrm{p} 2$ are the new momenta $\xi 1$ and $\xi 2$ are small displacements.

Then the Linearization of the Hamiltonian is done by substituting from Eqs. (16) into Equation (5), then the new states of the Hamiltonian in terms of new coordinates and new momenta can be written as:

$\mathrm{H}=\frac{1}{2}\left(\mathrm{p}^{2}+\mathrm{p}^{2}\right)+\mathrm{q}^{2} \mathrm{p} 1-\mathrm{q} 1 \mathrm{p} 2-V$

Now expand the potential function $V$ to second-order terms

$\mathrm{V}=\mathrm{V}(\mathrm{q} 1, \mathrm{q} 2)+V_{\mathrm{x}} \mathrm{q} 1+\mathrm{V}_{\mathrm{y}} \mathrm{q} 2+$

$+\frac{1}{2}\left(v_{x x} \mathrm{q}^{2}+2 v_{\mathrm{xy}} q 1 \mathrm{q}^{2}+\mathrm{v}_{\mathrm{yy}} \mathrm{q} 2^{2}\right)$

Where $\mathrm{q} 1$ and $\mathrm{q} 2$ are the coordinates of the libration point. The linear terms tend to zero because it is near to equilibrium points and the constant term does not affect the equation of motion. Then,

$$
\mathrm{V} \approx \frac{1}{2}\left(\mathrm{v}_{\mathrm{xx}} q 1^{2}+2 \mathrm{~V}_{\mathrm{xy}} \boldsymbol{q} 1 \mathrm{q} 2+\mathrm{v}_{\mathrm{yy}} \mathrm{q} 2^{2}\right)
$$

So,

$$
\mathrm{q} 1=\dot{\mathrm{x}}=\frac{\partial \mathrm{H}}{\partial \mathrm{q} 1}=-\mathrm{p} 2-\frac{1}{2}\left(2 \mathrm{~V}_{\mathrm{xx}} \mathrm{q} 1+2 \mathrm{~V}_{\mathrm{xy}} \mathrm{q} 2\right)
$$

$\dot{q} 2=\dot{y}=\frac{\partial H}{\partial q 2}=p 1-\frac{1}{2}\left(2 V_{x y} q 1+2 V_{y y} q 2\right)$

$$
\dot{\mathrm{p}} \mathbf{1}=\mathrm{p}_{\mathrm{x}}^{x}=\frac{\partial \mathrm{H}}{\partial \mathrm{p} 1}=\mathrm{p} 1+\mathrm{q} 2
$$


$\mathrm{p}^{2}=\mathrm{p}_{\mathrm{y}}^{*}=\frac{\partial \mathrm{H}}{\partial \mathrm{p} 2}=\mathrm{p} 2-\mathrm{q} 1$

Then from Eqs. (14), (15) and (20)

$\left[\begin{array}{c}q \dot{1} 1 \\ q \dot{q} \\ p 1 \\ p \dot{1} 2\end{array}\right]=J \nabla H_{2}=\left[\begin{array}{cccc}0 & 0 & 0 & 0 \\ 0 & 0 & 0 & 0 \\ -1 & 0 & 1 & 0 \\ 0 & -1 & 0 & 1\end{array}\right]\left[\begin{array}{c}-p 2-\left(v_{x x} q 1+v_{x y} q 2\right) \\ p 1-\frac{1}{2}\left(2 v_{x y} q 1+2 v_{y y} q 2\right) \\ p 1+q 2 \\ p 2-q 1\end{array}\right]$

This is reduced to:

$$
\left[\begin{array}{l}
q 1 \\
q= \\
q 2 \\
\dot{p} 1 \\
p 2
\end{array}\right]=\left[\begin{array}{cccc}
0 & 1 & 1 & 0 \\
-1 & 0 & 0 & 1 \\
v_{x x} & v_{x y} & 0 & 1 \\
v_{x y} & v_{y y} & -1 & 0
\end{array}\right]\left[\begin{array}{l}
q 1 \\
q 2 \\
p 1 \\
p 2
\end{array}\right]
$$

Then the auxiliary equation for the linearized system is given by:

$\lambda^{4}-\left(4-v_{x x}-v_{y y}\right) \lambda^{2}+V_{x x} v_{y y}-v_{x y}^{2}=0$

Eq. (23) has four roots which imply the behavior of the motion about the libration point.

\section{Lyapunov Analysis of Stability:}

At first, the potential must be positive definite for all $\mathrm{x}$ belongs to the domain about the libration point. Therefore, for asymptotic stability $\dot{\mathrm{V}}(\mathrm{x})<0$ for $\mathrm{x} \neq 0$ is required. Then, $V(x)$ is called a Lyapunov Function candidate and the system is stable in the sense of Lyapunov. Consider the potential defined by Eq. (19) at the collinear libration point, then $y=$ 0 . It is needed to find a quadratic function as:

$\mathrm{V}(\mathrm{x})=\mathrm{x}^{\mathrm{T}} \mathrm{P} \boldsymbol{x}$

Where

$\mathrm{P}=\mathrm{P}^{\mathrm{T}}>\mathbf{0}$

Then

$$
\dot{\mathrm{V}}(\mathrm{x})=\mathrm{x}^{\mathrm{T}} P \mathrm{x}+\mathrm{x}^{\mathrm{T}} \mathrm{P} \dot{\mathrm{x}}=-\mathrm{x}^{\mathrm{T}} \mathrm{Q} \boldsymbol{x}
$$

if there exists $Q=Q^{T}>0$ such that

$$
\mathrm{A}^{\mathrm{T}} \boldsymbol{P}+\boldsymbol{P A}=-\mathrm{Q}
$$

Where

$$
\mathrm{A}=\left.\frac{\partial \mathrm{V}}{\partial \mathrm{x}}\right|_{x=0}
$$

the scalar function $V(x)$ is positive definite, in which it is necessary $\dot{V}(\mathrm{x}) \leq 0$. Then, the origin is Lyapunov stable. Then, Lyapunov's center theorem is used to examine the stability of each of the libration points obtained.

\section{RESULTS AND DISCUSSION}

To apply our study the Sun-Earth-Moon system is considered, where the masses of the Earth, the Moon and the Sun in the canonical system are given as

$$
\begin{aligned}
& \mu 1=\frac{m_{1}}{m_{1}+m_{2}}=0.987871, \mu 2=\frac{m_{2}}{m_{1}+m_{2}}= \\
& 0.012151 \text { and } \mu 3=\frac{m_{3}}{m_{1}+m_{2}}=328900.48
\end{aligned}
$$

respectively, where the mass of the Earth $\mathrm{m} 1$, the mass of the Moon m2, and mass of the Sun

$$
\mathrm{M} 3=1.99 \times 10^{30} \mathrm{Kg} \text {. We take }
$$
$\mathrm{m} 1=1-\mu ; \mathrm{m} 2=\mu_{s}$ where $\mu$ is the mass ratio of the two primaries, and the unit distance between the two primaries is equal unity [19] and [20]. A code is constructed using Mathematica to obtain the libration point to prove that the potential of the system is Lyapunov function and is positive definite, finally to find the periodic orbits about this libration points and the Poincare maps.

The results obtained illustrate that there are five collinear libration points and three noncollinear libration points as shown in Table 1. For simplicity, the collinear libration point L2 and the non-collinear libration point L6 will be represented to illustrate a sample of the results obtained.

Table 1: the locations of the libration points in dimensionless coordinates

\begin{tabular}{|c|c|c|}
\hline Libration point & $\mathrm{x}$ & $\mathrm{Y}$ \\
\hline L1 & -1.85666 & 0 \\
\hline L2 & 1.05907 & 0 \\
\hline L3 & 0.908886 & 0 \\
\hline L4 & -0.902193 & 0 \\
\hline L5 & 0.597171 & 0 \\
\hline L6 & 1.06663 & 68.7966 \\
\hline L7 & 2.34696 & 68.7966 \\
\hline L8 & 417.112 & 68.7966 \\
\hline
\end{tabular}




\section{For the Libration Point L2 (1.05,0)}

The potential at the libration point is found to be positive and definite. The Lyapunov center theorem holds. Moreover, there are isolated periodic solutions emanating from this libration point which specified by the Poincare map. As shown in figures 2, 3, 4, and 5 the periodic orbits in the plane $(\mathrm{x}, \mathrm{x}, \mathrm{y})$, the Lyapunov orbits in $(\mathrm{x}, \mathrm{y})$ plane, the phase space, the Poincare map about L2 respectively.

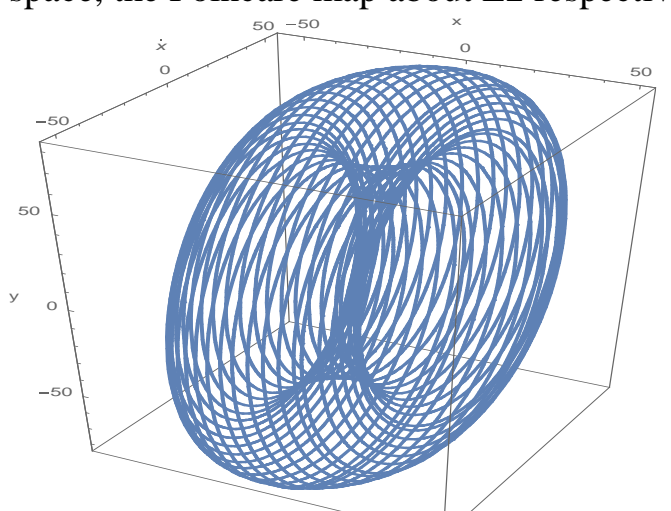

Fig. 2: The tree dimension periodic orbits about L2.

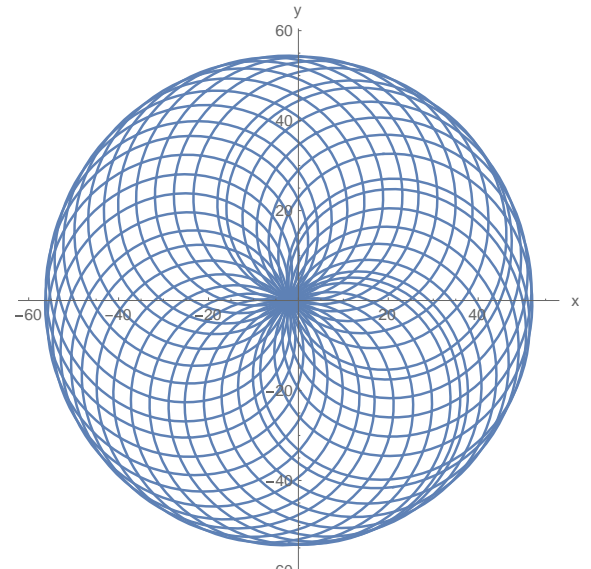

Fig. 3: the Lyapunov orbits about L2

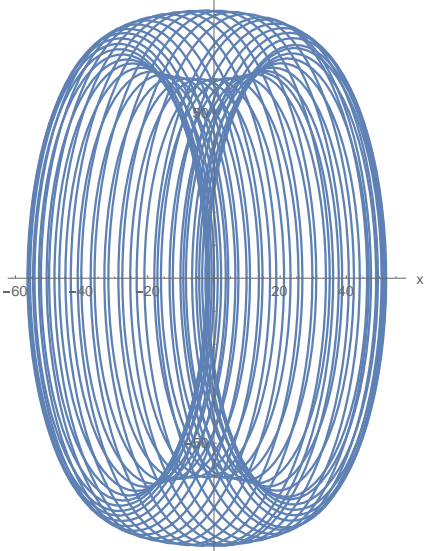

Fig. 4: the phase space about L2
For L6 (1.06663, 68.7966)

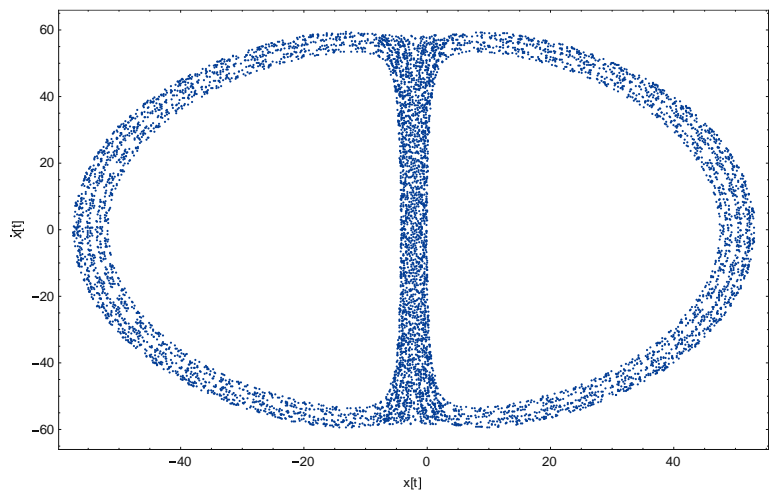

Fig. 5: the Poincare map about L2

The same is done and the results are illustrated in figures $6,7,8$, and 9 respectively.

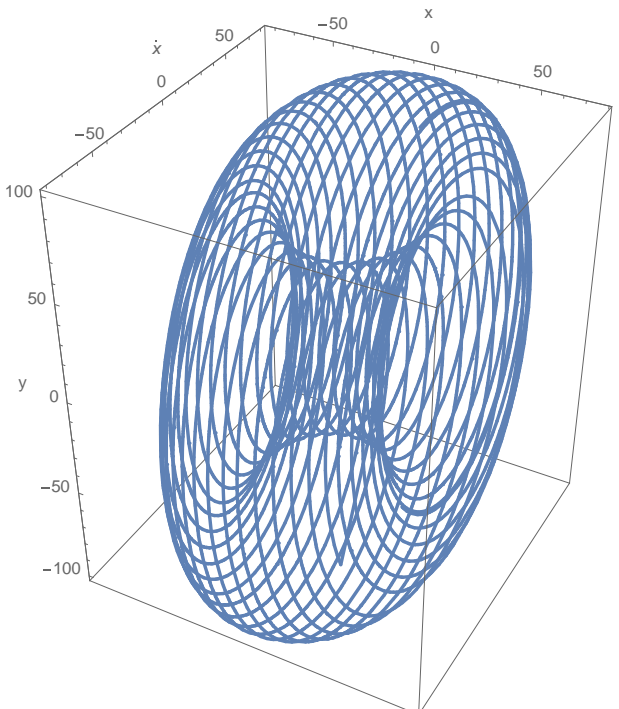

Fig. 6: The tree dimension periodic orbits about L6

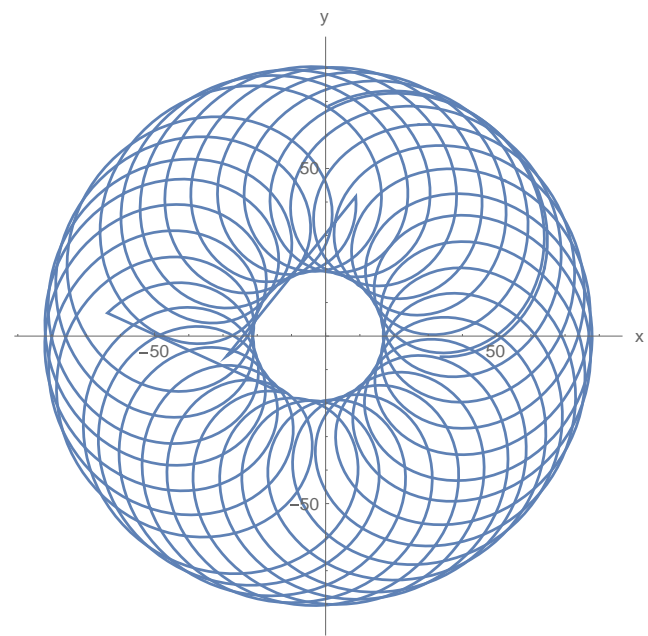

Fig. 7: the Lyapunov orbits about L6 


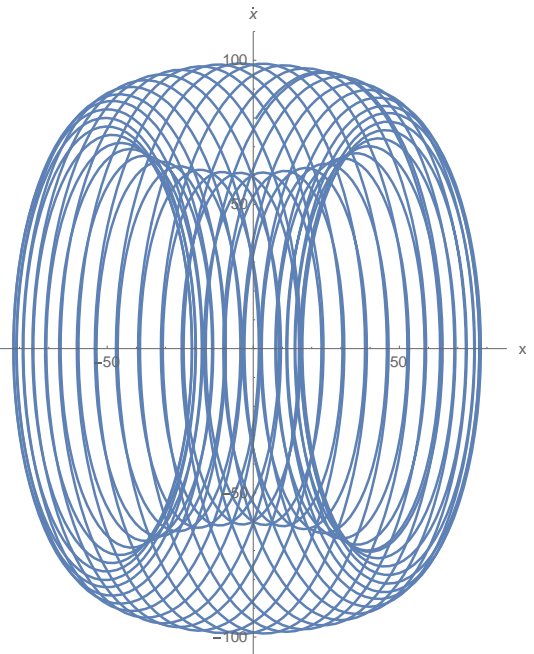

Fig. 8: the phase space about L6

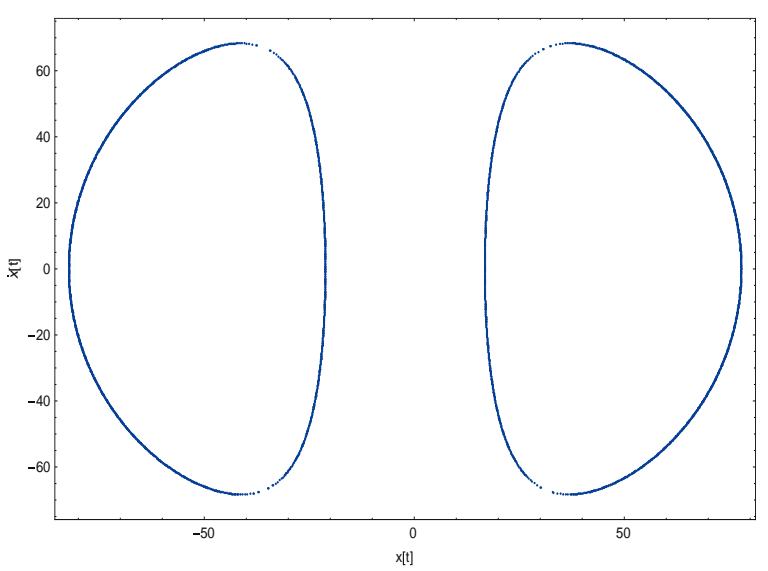

Fig. 9: the Poincare map about L6

\section{CONCLUSION}

Through this work, the four-body problem under the effects of the radiation and oblatness is studied. We observe that the locations of the libration points are shifted compared with those obtained by [20] who found the libration points of the four-body problem under gravitational and radiation only. The stability by applying the Lyapunov center theorem gives a good agreement with the Poincare map. Future work in the elliptic four-body problem will be a good field to be studied.

\section{REFERENCES}

[1] Gómez, G., Llibre, J., Martínez, R., \&Simó, C1987," Study on orbits near the triangular libration points in the perturbed restricted three-body problem", Final Report Fundacio Empresai Ciencia, Barcelona (Spain).

[2] Jorba, A.2000, "A numerical study on the existence of stable motions near the triangular points of the real Earth-Moon system, A dynamical systems approach to the existence of Trojan motions", Astronomy and Astrophysics, 364, 327-338.

[3] Gabern, F., \&Jorba, A. 2001, "A restricted fourbody model for the dynamics near the Lagrangian points of the Sun-Jupiter system", Discrete and Continuous Dynamical Systems Series B, 1(2), 143-182.

[4] Bell, J,1991," The impact of solar radiation pressure on Sun-Earth L1 libration point orbits", M. S. Thesis, Purdue University, West Lafayette, Indiana, August

[5] Guzman, J 2001, "Spacecraft Trajectory Design in The Context A Coherent Restricted FourBody Problem", Ph.D. Dissertation Purdue University.

[6] Pernicka, H, J 1990," The Numerical Determination of Nominal Libration Point Trajectories and Development of A StationKeeping Strategy", Ph.D. Dissertation Purdue University.

[7] Andreu, M, A 1998," The quasi-bicircular problem", Ph.D. Dissertation, University of Barcelona.

[8] Scheeres, D. 1998," The restricted Hill fourbody problem with applications to the EarthMoon-Sun system. Celestial Mechanics and Dynamical Astronomy, 70(2), 75-98.

[9] Baltagiannis, A. N., \& Papadakis, K. E 2011," Equilibrium points and their stability in the restricted four-body problem", International Journal of Bifurcation and Chaos, 21(08), 2179-2193.

[10] Baltagiannis, A. N., \& Papadakis, K. E.2011," Families of periodic orbits in the restricted four-body problem", Astrophysics and Space Science, 336(2), 357-367.

[11] Burgos-Garcia, J 2013," Regularization in the Restricted Four Body Problem", arXiv preprint arXiv:1310.5274.

[12] Burgos-García, J., \& Delgado, J 2013," Periodic orbits in the restricted four-body problem with two equal masses", Astrophysics and Space Science, 345(2), 247-263.

[13] Schechter, H. B 1968.'Three-dimensional nonlinear stability analysis of the sunperturbed earth-moon equilateral points", AIAA Journal, 6(7), 1223-1228.

[14] Carpenter, L., \& Kolenkiewicz, R 1968," Stable periodic orbits about the sun perturbed earth-moon triangular points", AIAA Journal, 6(7), 1301-1304.

[15] Dutt, P., \& Sharma, R. K 2010," Analysis of periodic and quasi-periodic orbits in the EarthMoon system", Journal of guidance, control, and dynamics, 33(3), 1010-1017.

[16] Dutt, P., \& Sharma, R. K2012, "On the evolution of the ' $\mathrm{f}$ ' family in the restricted 
three-body problem. Astrophysics and Space Science, 340(1), 63-70.

[17] Ibrahim, A.H., Ismail, M.N., Zaghrout, A.S., Younis, S.H., and El Shikh, M.O\{ 2018), "Lissajous Orbits at the Collinear Libration Points in the Restricted Three-Body Problem with Ob-lateness", World Journal of Mechanics, 8, 242-252.

[18] Kumari, R., \&Kushvah, B. S. (2013). Equilibrium points and zero velocity surfaces in the restricted four-body problem with solar wind drag. Astrophysics and Space Science, 344(2), 347-359.

[19] Ismail, M. N., Younis, S. H., \&Elmalky, F. M. (2018). Modeling sun's radiation effect on restricted four bodies. NRIAG Journal of Astronomy and Geophysics, 7(2), 208213.

[20] Ismail, M. N., Ibrahim, A. H., Zaghrout, A. S., Younis, S. H., Elmalky, F. S., \&EImasry, L. E. (2019). Solar Radiation Pressure Effects on Stability of Periodic Orbits in Restricted Four-Body Problem. World Journal of Mechanics, 9(08), 191.

[21] Sengupta, P., \& Singla, P. (2002). An Analysis of Stability in the Restricted Three-Body Problem.

[22] Zwillinger, D. (1998). Handbook of differential equations (Vol. 1). Gulf Professional
Publishing.

[23] Lynch, S. (2007). Dynamical systems with applications using Mathematica ${ }^{\circledR}(p p .111$ 123). Boston: Birkhäuser.

[24] Cottle R. W., Habetler G. J., \& Lemke C. E. (1967). QUADRATIC FORMS SEMIDEFINITE OVER CONVEX CONES (No. TR-67-6). STANFORD UNIV CALIF OPERATIONS RESEARCH HOUSE.

[25] Gantmacher F. R., \& Brenner J. L. (2005). Applications of the Theory of Matrices. Courier Corporation.

[26] GREGORY R. AND D. KARNEY D. (1969). A Collection of Matrices for Testing Computational Algorithms, WileyInterscience, New York, 1969.

[27] HORN R. A. AND C. R. JOHNSON C. R. (1994).Topics in Matrix Analysis, Cambridge University Press, Cambridge.

[28] GAJI'C AND M. QURESHI (2008). Lyapunov Matrix Equation in System Stability and Control, Dover, New York,

[29] HAMMARLING S. J. (1982). Numerical solution of the stable, nonnegative definite Lyapunov equation, IMA J. Numer.Anal., 2 (1982), pp. 303-323.

[30] Rantzer A. (2001). A dual to Lyapunov's stability theorem. Systems \& Control Letters, 42(3), 161-168.

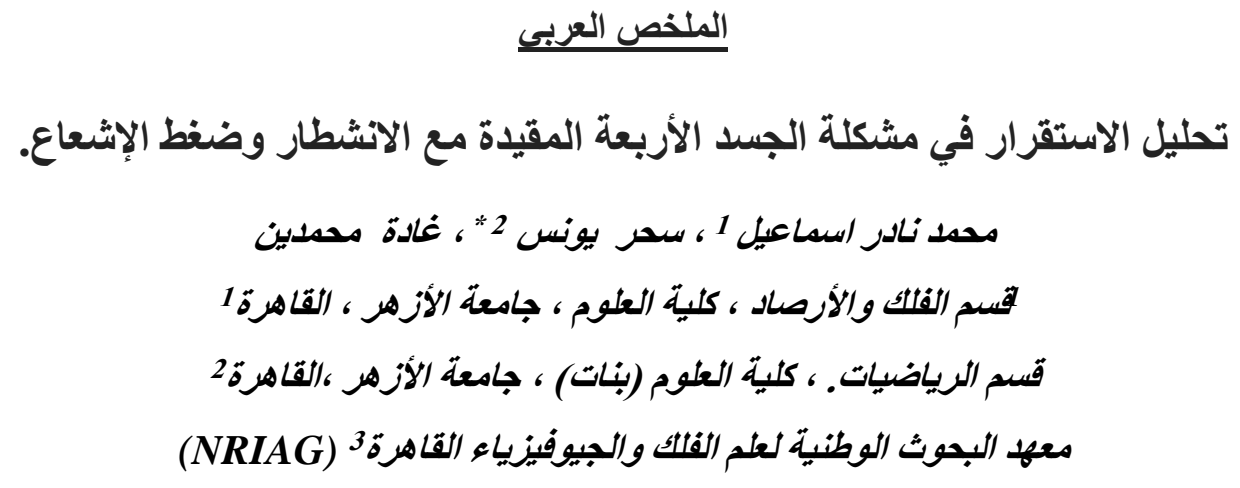

في العمل الحالي ، يُستمد الثكل القانوني للمعادلات التفاضلية من دالة هاميلتونيان التي يتم الحصول عليها لنظام

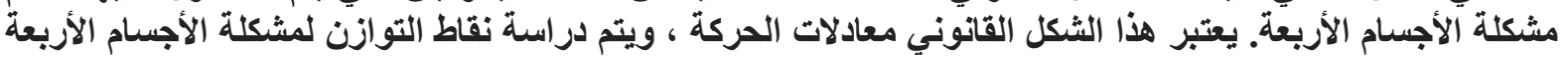

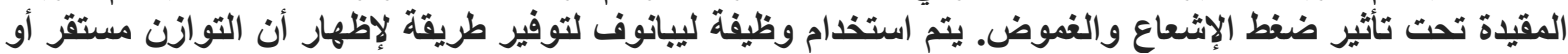

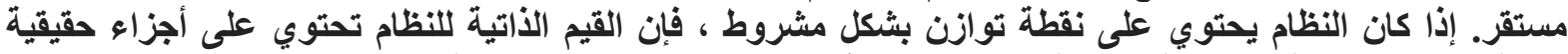

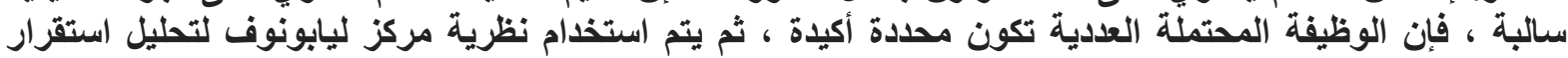

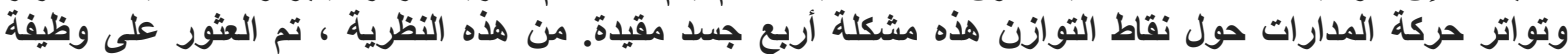

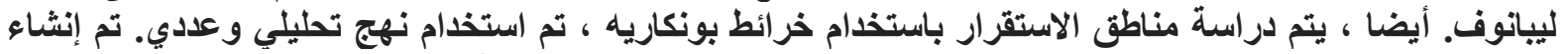

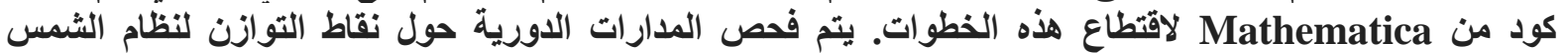
والأرض والقنمر. 